\title{
Effects of social conditions and time of testing on activity and striking of goldfish (Carassius auratus)*
}

\author{
RICHARD H. BAUER and JAMES H. TURNER \\ University of Houston, Houston, Texas 77004
}

\begin{abstract}
Pairs of goldfish were separated by a clear Plexiglas sheet, an opaque sheet, or mirrors with the reflective surfaces toward the fish. Horizontal activity, vertical activity, and striking for food pellets were recorded at 7:00 a.m., 12:00 noon, and 4:00 p.m. for 14 days. Horizontal and vertical activity was greater at 12:00 and 4:00 than at 7:00, increased for approximately the first 8 days, and then declined slightly. By the 10th day, horizontal activity was greater for fish with mirror-image stimulation and those separated by a clear Plexiglas sheet than fish separated by an opaque sheet. Striking for food pellets was not altered by social conditions or time of testing. The results suggest that social facilitation of feeding is not related to increased activity, but probably requires competition for food.
\end{abstract}

Use of the goldfish (Carassius auratus) in psychological and physiological research has increased dramatically in the past several years. Although laboratory conditions are known to influence behavior of other species, relatively little is known about those conditions which influence behavior of the goldfish. Since presence of another conspecific in the testing situation and time of day Ss are tested alter activity of rodents (e.g., Archer, 1969; Hughes, 1969), the present study examined the effects of these variables on activity of goldfish.

Feeding behavior of a number of species, including fish, is also influenced by social conditions (Tolman, 1968; Welty, 1934; Zajonc, 1965). In general, more food is eaten when animals are tested in the presence of another conspecific than when tested in isolation. Zajonc (1965) suggested that social facilitation effects are due to an increase in general arousal or activity. Therefore, those conditions resulting in greater activity would be expected to facilitate feeding. Striking of food pellets was also examined to determine if social facilitation of feeding and activity were related.

\section{METHOD}

\section{Subjects}

The Ss were 21 experimentally naive goldfish, $7.6-10.2 \mathrm{~cm}$ in body length, obtained from Ozark Fisheries, Stoutland, Missouri. The fish were maintained in the laboratory for 14 days before the experiment. Maintenance conditions were identical to those described by Braud (1970).

\section{Apparatus}

The aquariums were $19.0 \mathrm{~cm}$ high, $20.5 \mathrm{~cm}$ in diam, and constructed of clear plastic. Gravel was placed in the tank bottoms, and each tank contained approximately two gallons $(17.0 \mathrm{~cm})$ of water. Water temperature was maintained at $72^{\circ} \mathrm{F}$. Oxygen was provided to the tanks by aerators. Each tank was

*This paper is sponsored by Neal Grossen, who takes full editorial responsibility for it. The research was supported by National Institute of Mental Health Grant MH-243 84-01 to Joaquin Fuster and Richard $H$. Bauer, who is now in the Department of Psychiatry, School of Medicine, University of California, Los Angeles, California 90024. partially wrapped with opaque paper to prevent any vision between the tanks. The tanks were separated at the center by (a) a 0.5 -cm-thick clear Plexiglas sheet, (b) a 0.5 -cm-thick flat brown opaque sheet which prevented all vision between the two halves, or (c) two mirrors with the reflective surfaces facing outward.

\section{Procedure}

One fish was randomly assigned to each half of the aquarium $(\mathrm{N}=7$ per group). Ss were adapted to the aquarium for 2 days prior to the start of the experiment. On the third day, measures of horizontal and vertical movement were recorded, starting at 7:00 a.m., 12:00 noon, and 4:00 p.m. A horizontal movement was recorded each time the mouth of the fish crossed a line dividing his portion of the tank. Vertical movement was recorded each time the fish's mouth crossed a line dividing the water level in half. Activity of each fish was recorded for $2 \mathrm{~min}$, and order of testing was randomized for each session.

Immediately after each session, the fish were fed one Shrimp-el-ett pellet. The pellet was dropped into the tank from approximately $8.0 \mathrm{~cm}$ above the water and the number of strikes recorded. A strike was defined as any effort by the fish to strike the pellet before it reached the tank bottom.

Lighting in the experimental room was provided by a combination of natural and artificial lighting. Mean sunrise and sunset during the experimental period were approximately 7:00 a.m. and 6:30 p.m. The room light was turned on $5 \mathrm{~min}$ before each morning session and turned off immediately after the last afternoon session.

\section{RESULTS}

Preliminary analysis showed that type of social condition did not significantly alter vertical or horizontal activity. Therefore, type of social condition was not considered further.

The upper panel of Fig. 1 presents mean vertical crossings per minute at 7:00 a.m., 12:00 noon, and 4:00 p.m. in blocks of 2 days. As can be seen, vertical activity appears to be greater at $12: 00$ and $4: 00$ p.m. than at 7:00 a.m., and increased for the first 6 days and then declined. Number of crossings in the vertical and horizontal direction were analyzed separately by a 3 by 3 by 7 complete factorial analysis of variance. The main factors were type of social conditions, time of day, and 


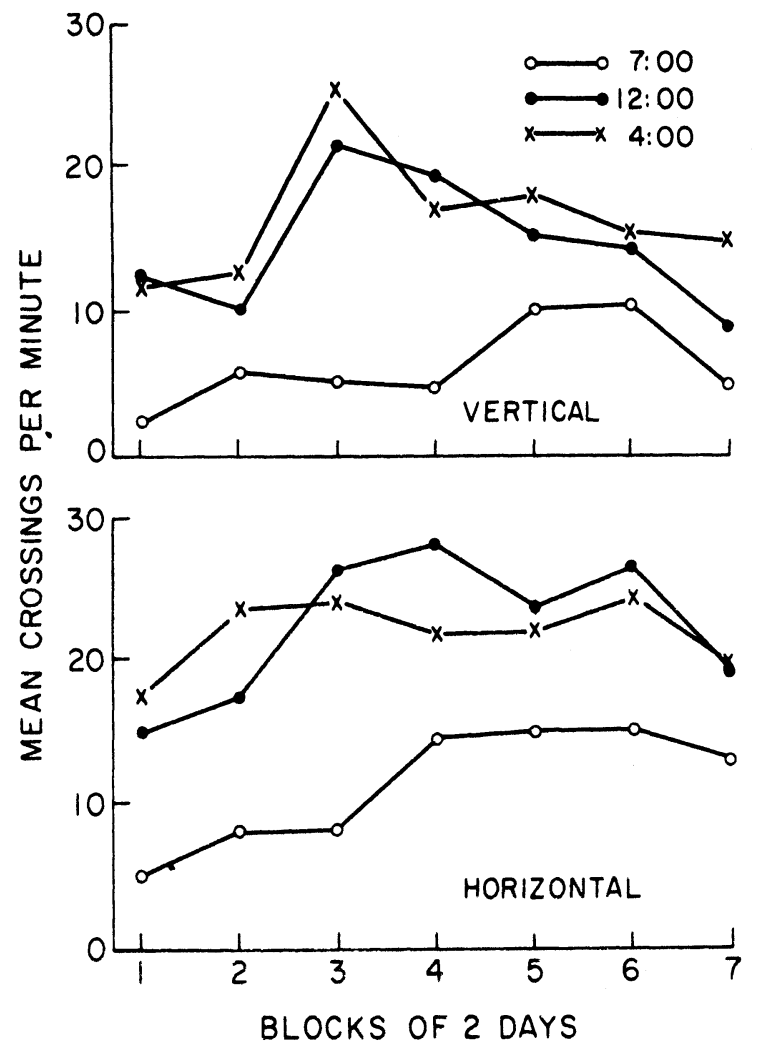

Fig. 1. Mean vertical and horizontal crossings per minute at 7:00 a.m., 12:00 noon, and 4:00 p.m. in blocks of 2 days.

blocks of 2 days. The last two factors were treated as repeated measures factors. Analysis of crossings in the vertical direction revealed that the main effects for time of day, $F(2,36)=20.75, p<.001$, and days, $F(6,108)=$ $3.15, \mathrm{p}<.007$, were significant. Comparisons between individual treatment means by Duncan multiple range tests indicated that vertical activity was significantly greater at 12:00 and 4:00 than at 7:00 (p<.01).

The lower panel of Fig. 1 shows mean crossings per minute in the horizontal direction. Similar to vertical activity, horizontal activity appears greater at 12:00 and 4:00 than at 7:00. Analysis of horizontal crossings indicated that the main effects for time of day, $F(2,36)$ $=31.10, \mathrm{p}<.001$, and days, $\mathrm{F}(6,108)=2.91, \mathrm{p}<.02$, were significant. The Time of Day by Days interaction was also significant, $\mathrm{F}(12,216)=2.22, \mathrm{p}<.02$. Individual comparisons by Duncan's multiple range test indicated that horizontal activity was greater at 12:00 and 4:00 than at 7:00 $(\mathrm{p}<.01)$. Although there was no significant main effect for the type of social conditions, $F(2,18)=0.88$, the Social Condition by Days interaction was significant, $\mathrm{F}(12,108)=1.96, \mathrm{p}<.03$. The significant interaction between social condition and days was due to the fact that horizontal activity of fish with mirror-image stimulation and the clear partition increased across days, while activity of fish with the opaque partition decreased slightly across days.
Analysis of variance of the number of strikes indicated that the effects for days and time of day were nonsignificant. Although fish with mirror-image stimulation struck more often $(82 \%)$ than those with the clear $(71 \%)$ or opaque $(69 \%)$ partitions, there was no significant difference between the groups, $F(2,18)=$ 0.73 .

\section{DISCUSSION}

Previous investigators have reported that the presence of another conspecific in the testing situation can lead to increased general activity (Hughes, 1969; Zajonc, 1965). However, previous studies on the effects of preexperimental grouping and isolation on activity of rodents are in conflict (e.g., Archer, 1969). Archer found that the duration of isolation or grouping was an important parameter in determining the degree of activity and suggested that the duration variable could account for these conflicting results. In the present study, there were no initial activity differences between the groups, but by the 10th day, horizontal activity of fish with mirror-image stimulation and the clear partition was twice that of fish with the opaque partition. Therefore, longer exposure to the present conditions may result in a further activity increase by fish with mirror-image stimulation and separated by a clear partition and a decrease by fish separated by an opaque partition. Mirror-image stimulation would be expected to increase activity of goldfish because goldfish prefer mirror-image stimulation to another conspecific behind Plexiglas (Gallup \& Hess, 1971). Gallup, Montevecchi, \& Swanson (1972) have also suggested that mirror-image stimulation is a supernormal social stimulus for some species.

Shashoua (1973) recently reported that goldfish exhibit annual changes in their learning and activity patterns, with periods of low activity coinciding with poor learning. Activity of other species changes throughout the day and is related to avoidance conditioning (e.g., Davis, Navaratnam, \& Redfern, 1973). Since goldfish in the present study were more active at 12:00 and 4:00 than at 7:00, the time of day goldfish are trained may facilitate or disrupt learned behaviors, depending upon requirements of the task.

Although goldfish in the present study showed activity differences as a function of time of day and social conditions, increased activity was not associated with increased striking for food. Therefore, the hypothesis that social facilitation of feeding is due to increased activity or arousal is not supported (Zajonc, 1965). Tolman (1964) has suggested that competition is necessary for facilitation of eating. In support of this hypothesis, goldf ish are reported to eat more when in competition for food than when fed in isolation (Welty, 1934). Since fish in the present study were not in competition for food and did not differ in their striking behavior, the results lend support to the hypothesis that competition is a necessary requirement for social facilitation of feeding in the goldfish.

\section{REFERENCES}

Archer, J. Contrasting effects of group housing and isolation on subsequent open field exploration in laboratory rats. Psychonomic Science, 1969, 14, 234-235.

Braud, W. G. Extinction in goldfish: Facilitation by intracranial injection of RNA from brains of extinguished donors. Science, 1970, 139, 1234-1236.

Davis, J. A., Navaratnam, V., \& Redfern, P. H. A 24-hour rhythm in passive-avoidance behavior in rats. Psychopharmacologia, 1973, 32, 211-214.

Gallup, G. G., Jr., \& Hess, J. Y. Preference for mirror-image stimulation in goldfish (Carassius auratus). Psychonomic Science, 1971, 23, 63-64. 
Gallup, G. G., Jr., Montevecchi, W. A., \& Swanson, E. T. Motivational properties of mirror-image stimulation in the domestic chicken. The Psychological Record, 1972, 22, 193-199.

Hughes, R. N. Social facilitation of locomotion and exploration in rats. British Journal of Psychology, 1969, 60, 385-388.

Shashoua, V. E. Seasonal changes in the learning and activity patterns of goldfish. Science, 1973, 181, 572-574.
Tolman, C. W. Social facilitation of feeding behavior in the domestic chick. Animal Behaviour, 1964, 12, 245-251.

Welty, J D Experiments in group behavior of fishes. Physiological Zoology, 1934, 7, 85-128.

Zajonc, R. B. Social facilitation. Science, 1965, 149, 269-274.

\title{
On the establishment of a continuous repertoire*
}

\author{
SHEILA CHASE, ETHEL A. GELLER, and JEAN S. HENDRY \\ Hunter College of the City University of New York, New York, New York 10021
}

Pigeons trained to peck the response key directly below a spot of light which appeared in only three of five positions were tested with the spot in all five positions. Key choice, given these test stimuli, showed a mixture of functional control: on some trials, the key beneath the spot was chosen, indicating that the training produced a correspondence between novel points on the stimulus and response dimensions (a continuous repertoire); on other trials, the specific responses acquired during training were made.

Though behavior is often analyzed into discrete responses to discrete stimuli, much of it in fact consists of continuously variable activity related to continuous variations in stimulation. For example, to reach a ledge, a pigeon must adjust its flight pattern continuously in response to changing stimulation; similarly, to avoid a collision, a driver must adjust the position of the steering wheel in response to continuously changing stimulation. In situations of this sort, both stimuli and responses vary

*Reprints may be obtained from S. Chase, Department of Psychology, Hunter College, 695 Park Avenue, New York, New York 10021. along one or more continua; in the terminology of Skinner (1953), they comprise continuous fields. The functional control of behavior may be described by specifying the correspondence between points in the stimulus and response fields.

Some correspondences of this sort are surely the product of learning; however, the learning processes involved are very poorly understood. Since it is unreasonable to assume that all possible combinations of stimuli and responses have been encountered during prior learning experiences, some psychologists have assumed that the correspondence comes about through a process of extrapolation based on training during which 